\title{
Evaluación del método ELISA de punto para el diagnóstico de la cisticercosis humana y para estimar valores de prevalencia en una región endémica en Colombia
}

\author{
Piedad Agudelo ${ }^{1}$, David Botero ${ }^{1}$ Luis Guillermo Palacio ${ }^{2}$ \\ ${ }^{1}$ Instituto Colombiano de Medicina Tropical-Instituto de Ciencias de la Salud, Medellín, Colombia. \\ ${ }^{2}$ Instituto Neurológico de Antioquia, Medellín, Colombia.
}

Introducción. La cisticercosis continúa siendo un problema de salud pública a nivel mundial. El diagnóstico de esta enfermedad se hace por medio de la detección de anticuerpos específicos y de técnicas de imaginología.

Objetivo. Evaluar la ELISA de punto, método inmunoenzimático, en la detección de anticuerpos contra Taenia solium para ser usado tanto en pacientes con neurocisticercosis como en poblaciones donde es endémica.

Materiales y métodos. Se utilizaron 45 muestras de suero, 41 de plasma y 23 de líquido cefalorraquídeo de pacientes con cisticercosis confirmada según criterios clínicos, quirúrgicos, imaginológicos y de laboratorio. Además, se estudiaron 37 muestras de suero, 64 de plasma y 17 de líquido cefalorraquídeo de personas que no presentaban cisticercosis y que no tenían antecedentes epidemiológicos de teniosis-cisticercosis. Se evaluaron también 43 muestras de suero de personas con parasitosis diferentes a cisticercosis y 663 muestras de suero de un estudio de seroprevalencia de cisticercosis en una comunidad rural colombiana. Las muestras se procesaron tanto por inmunoelectrotransferencia como por la ELISA de punto. La inmunoelectrotransferencia se utilizó como prueba de oro para establecer la sensibilidad y la especificidad de la ELISA de punto. Se analizaron 933 muestras.

Resultados. Con las 109 muestras de los individuos afectados por cisticercosis y las 118 muestras de controles sanos se hicieron los análisis estadísticos de validación de la prueba diagnóstica y se obtuvo para la ELISA de punto una sensibilidad total de 80,7\% (IC95\%: 80,2\% a $81,2 \%$ ) y una especificidad de $92,4 \%$ (IC95\%: $91,9 \%$ a $92,8 \%$ ). La ELISA de punto realizado con suero, plasma y líquido cefalorraquídeo tuvo una sensibilidad de $91,1 \%, 85,4 \%$ y $52,12 \%$, respectivamente. La misma prueba evaluada en suero, plasma y líquido cefalorraquídeo tuvo una especificidad de $100 \%$, 85,9\% y $100 \%$, respectivamente. Las 43 muestras de personas con otras parasitosis diferentes de cisticercosis fueron negativas para ambas pruebas.

Cuando la ELISA de punto se utilizó para el diagnóstico de la cisticercosis en una población endémica colombiana en la que 1,81\% (12/663) de las personas presentó anticuerpos para $T$. solium por inmunoelectrotransferencia, la ELISA de punto presentó una sensibilidad de 58,3\% (IC95\%: 54,0\% a 62,7\%) y una especificidad de 100\% (IC95\%: 99,9\% a 100,0\%) con un valor diagnóstico positivo de 100\% y un valor diagnóstico negativo de 99,2\%.

Conclusión. La ELISA de punto es una prueba promisoria como herramienta diagnóstica en estudios epidemiológicos de tamizaje.

Palabras clave: test de ELISA, western blot, Taenia solium, cisticercosis.

Evaluation of the ELISA method for diagnosis of human cysticercosis in an endemic region

Introduction. Cysticercosis is a worldwide public health problem. Currently it is diagnosed by detection of specific antibodies or by imaging techniques.

Objective. To evaluate an alternative diagnostic tool, a simple antibody detection assay, called Dot ELISA, for immunological diagnosis of patients with neurocysticercosis as well as for endemic population screening.

Materials and methods. The test was applied to cysticercosis patients, as well as to healthy 
controls and individuals with other parasitic infections. A total of 45 serum, 41 plasma and 23 cerebrospinal fluid samples were obtained from patients meeting clinical, surgical, imaging and laboratory criteria for cysticercosis. Samples were processed by enzyme-linked immuneelectro-transfer blot assay and by Dot ELISA. Controls included 37 serum, 64 plasma and 17 cerebrospinal fluid samples from healthy individuals without epidemiological history for taeniosiscysticercosis. Similarly, 43 plasma samples from patients with parasitic infections different from cysticercosis and 663 samples from population survey for cysticercosis were also evaluated. Results. A total of 933 samples were analyzed. In samples from cysticercosis patients and healthy control individuals, the Dot ELISA test showed an overall sensitivity of $80.7 \%$ $(\mathrm{Cl} 95 \%=80.2 \%-81.2 \%)$ and a specificity of $92.4 \%(\mathrm{Cl} 95 \%=91.9 \%-92.8 \%)$. The Dot ELISA performed in serum had a sensitivity of $91.1 \%$, in plasma $85.4 \%$, and in cerebrospinal fluid $52.2 \%$. Similarly, the same test performed in serum, plasma and cerebrospinal fluid, had a specificity of $100 \%, 85.9 \%$ and $100 \%$ respectively. The Dot ELISA was applied as a screening test for the diagnosis of cysticercosis in an endemic population in which $1.8 \%(12 / 663)$ of individuals had T. solium antibodies detected by Enzyme-linked immune-electro-transfer blot assay and showed a sensitivity of $58.3 \%$ (CI95\% $54.0-62.7)$ and a specificity of $100 \%$ (CI95\% $99.9-100.0$ ) with a positive predictive value of $100 \%$ and a negative predictive value of $99.2 \%$. All 43 samples from patients with parasitic infections different from cysticercosis were negative for both tests.

Conclusions. These results indicated that Dot ELISA is a promising tool for the diagnosis of cysticercosis as a screening test, as well as for field epidemiological studies.

Key words: enzyme-linked immunosorbent assay, western blot, Taenia solium, cysticercosis.

La cisticercosis es una enfermedad parasitaria producida por la larva Taenia solium que afecta tanto al hombre como a los cerdos. Se adquiere al ingerir los huevos producidos por los adultos de este parásito. Debido a que el ser humano es el único hospedero definitivo del parásito adulto, esta parasitosis intestinal es el factor más importante de contaminación (1).

Tanto en el hombre como en el cerdo, los huevos se desarrollan en cisticercos y alcanzan diferentes localizaciones tisulares. El sistema nervioso central (SNC) es el sitio de localización más frecuente, y allí se presenta la neurocisticercosis humana (1).

Esta parasitosis, conocida desde la antigüedad, es la más frecuente del SNC. Es considerada como un problema de salud pública en los países

\author{
Correspondencia: \\ Piedad Agudelo-Flórez \\ Carrera 43A No 52 Sur-99 \\ Sabaneta, Antioquia, Colombia. A.A. 52162 \\ Teléfono 57-4-3053500 ext 314 \\ Fax 57-4-3014258 \\ pagudelo@ces.edu.co
}

Recibido:12/05/05; aceptado: 19/08/05 endémicos, y emerge como un importante problema de salud de dimensiones globales, debido a la migración de individuos con la parasitosis intestinal desde las regiones consideradas endémicas. La Organización Mundial de la Salud estima en 50.000 las muertes debidas a cisticercosis cada año en el mundo (2-5).

El diagnóstico de la cisticercosis se hace con base en la información clínica y epidemiológica, en pruebas imaginológicas como la tomografía axial computarizada (TC) y la resonancia magnética (RM), y en pruebas serológicas como el inmunoensayo enzimático (ELISA) y la inmunoelectrotransferencia (EITB). La prueba de ELISA permite la detección tanto de anticuerpos como de antígenos. El EITB es la prueba más sensible y específica de todas las de tipo inmunológico de las que se dispone actualmente (6-10).

Las pruebas diagnósticas, a pesar de lo efectivas que pueden ser, presentan dificultades técnicas porque requieren una compleja implementación que difícilmente esta disponible en centros de atención en salud de primero y segundo nivel, ya que requieren personal altamente capacitado y tecnología costosa. 
Por las razones expuestas, el presente estudio buscó evaluar una prueba serológica rápida y de fácil aplicación, el ELISA de punto (EP) o inmunopunto, para complementar el diagnóstico de la cisticercosis sin necesidad de tecnología costosa. Se evalúo también la muestra biológica más apropiada para llevar a cabo la prueba.

Una vez la prueba estuvo validada en condiciones de laboratorio, se determinó su sensibilidad y especificidad como método de tamizaje para la identificación de individuos con cisticercosis en una población endémica a través de un estudio epidemiológico.

\section{Materiales y métodos}

La recolección de muestras para la evaluación de la prueba se hizo en diferentes regiones de Antioquia y Chocó, departamentos localizados en la región noroccidental de Colombia. Se obtuvieron 933 muestras con las que se conformaron los siguientes grupos de estudio: a) 109 muestras de pacientes con diagnóstico de cisticercosis, de las cuales 45 fueron de suero, 41 de plasma y 23 de LCR; b) 118 muestras de personas negativas para cisticercosis que no presentaban antecedentes clínicos y epidemiológicos de teniosiscisticercosis. De ellas, 37 eran de suero, 64 de plasma y 17 de LCR; c) 43 muestras de suero de pacientes con parasitosis diferentes a cisticercosis, y d) 663 muestras de suero de un estudio de seroprevalencia de cisticercosis (10).

Las muestras positivas para cisticercosis fueron seleccionadas de acuerdo con criterios clínicos, quirúrgicos e imaginológicos; algunas fueron donadas por el Instituto Neurológico de Antioquia y otras remitidas para diagnóstico serológico al Instituto Colombiano de Medicina Tropical (ICMT). El diagnóstico clínico depende del número, localización y viabilidad de los quistes. Todos los pacientes positivos presentaron signos y síntomas de neurocisticercosis, especialmente epilepsia de inicio después de los 10 años de edad que fue confirmada mediante estudio imaginológico (resonancia magnética o escanografía) para detectar al menos dos quistes viables localizados en el parénquima cerebral. Algunas muestras pertenecían a pacientes que fueron intervenidos quirúrgicamente por presentar neurocisticercosis y en quienes se confirmó la presencia del quiste mediante estudio histopatológico.

Las muestras negativas para cisticercosis y las muestras positivas para parasitosis diferentes a cisticercosis, utilizadas para determinar reacciones cruzadas, fueron obtenidas en el laboratorio del ICMT. Todas las muestras fueron conservadas a $-20^{\circ} \mathrm{C}$ hasta su uso. Con el fin de determinar reacciones cruzadas con otras parasitosis se incluyeron 43 muestras de individuos con diagnósticos diferentes a cisticercosis, entre ellas dos muestras de teniosis intestinal por T. saginata, cuatro de Toxoplasma gondii, 15 de Strongyloides stercoralis, una de Dirofilaria sp., dos muestras de parasitosis intestinales múltiples (uncinariosis, entamebosis, estrongiloidosis y giardiosis), dos de Onchocerca volvulus, una de Brucella sp., una de Plasmodium sp. y 15 de Entamoeba histolytica.

Este proyecto fue aprobado por el Comité de Ética del ICMT, pues cumplía con las normas establecidas por el Ministerio de Salud de Colombia en lo referente a la investigación con personas y animales (Resolución № 008430 de 1993). De acuerdo con esta resolución, la investigación se consideró de riesgo mínimo. Se obtuvo consentimiento informado por escrito de todas las personas que participaron en la investigación.

Todas las muestras se analizaron con las dos pruebas referenciadas (EP y EITB).

\section{Desarrollo del EP}

Los antígenos se obtuvieron a partir de cisticercos de $T$. solium disecados del tejido muscular de dos cerdos infectados naturalmente y procedentes del departamento de Antioquia, Colombia. Luego de ser extraídos, los cisticercos fueron lavados con solución amortiguadora de fosfatos (PBS) pH 7,2 fría y se pesaron secos en papel de filtro dando un peso total de $150 \mathrm{gr}$. Posteriormente fueron almacenados a $-80^{\circ} \mathrm{C}$ hasta el día de su procesamiento.

\section{Protocolo para obtención del antígeno crudo}

Se utilizó la metodología descrita por Grogl et al. (11) que, en términos generales, consistió en el 
lavado de los cisticercos con PBS frío al que se le agregaron $2 \mathrm{mM}$ de fenilmetilsulfonilfluoruro (PMSF) como inhibidor enzimático. Después, se homogenizaron los cisticercos a $0^{\circ} \mathrm{C}$ durante 100 ciclos en un homogenizador de tejidos TEKMAR Cincinnati Ultra-Turrax ${ }^{\circledR}$ TR-10 Power Control. El material obtenido fue sometido a rompimiento con ultrasonido en hielo por 6 ciclos de 3 minutos, cada uno a $20 \mathrm{kHz}$, con 30 segundos de intervalo de enfriamiento, en un sonicador Sonifier ${ }^{\circledR}$ modelo 200. El producto resultante fue centrifugado a $25.000 \mathrm{~g}$ durante una hora a $4^{\circ} \mathrm{C}$ y el sobrenadante fue dializado contra agua bidestilada estéril y fría durante una noche, tras lo cual se hizo una medición preliminar de proteínas por el método de Bradford (12). El extracto antigénico fue distribuido en alícuotas y almacenado $\mathrm{a}-80^{\circ} \mathrm{C}$ hasta su uso. Este antígeno fue utilizado en la realización del EP.

\section{Desarrollo del EP}

El EP se efectuó siguiendo las consideraciones de Cardona y Agudelo (13). Para unir el antígeno, se utilizó como fase papel de nitrocelulosa (BioRad Laboratories, Cat No 162-0117, Richmond CA, USA); esta se prehumedeció en buffer salino fosfato (DPBS, D-5773,Sigma, St. Louis, MO, USA). Una vez pretratada, la membrana se colocó en un aparato Bio Dot Microfiltration Apparatus marca Bio Rad Laboratories (Richmond CA, USA, referencia 170-6545). La membrana se humedeció nuevamente con 100 uL por pozo de PBS y se hizo vacío para drenar los pozos, luego se fijó el antígeno a una concentración de $3 \mathrm{ug} / \mathrm{ml}$ en $100 \mathrm{ul}$ por pozo de solución amortiguadora de carbonatobicarbonato, pH 9,6. Este paso de fijación del antígeno a la membrana se llevó a cabo durante 2 horas. Ésta, y las demás incubaciones, se realizaron a temperatura ambiente y con agitación constante en un agitador rotatorio.

Posteriormente, se procedió a bloquear los sitios donde el antígeno no se había unido para evitar uniones inespecíficas, utilizando una solución de PBS y albúmina sérica bovina al 1\% (BSA, Sigma, St. Louis, MO, USA) y polioxietilensorbitanomonolaurato Tween ${ }^{\circledR} 20$ (Merck, Schuchardt, 8011 ) al $0,05 \%$. Este proceso se llevó a cabo durante una hora.
Luego se procedió a realizar tres lavados consecutivos con PBS-Tween $\AA 20$ al 0,05\%, después de los cuales se adicionaron las muestras a ser evaluadas a las diferentes concentraciones determinadas en la estandarización, cuidando de que hubiera pozos blanco, muestras negativas y muestras positivas en cada lote procesado, con el propósito de realizar el control de calidad de la prueba. La concentración para las muestras de suero y plasma fue de 1:50 y para LCR de 1:10, y el tiempo de incubación fue de una hora.

Después de realizar un nuevo lavado con la solución ya señalada, se adicionó el conjugado previamente titulado de anti-inmunoglobulina $G$ anti-humana marcada con peroxidasa (A-8792, Sigma St. Louis, MO, USA), a una concentración de 1:2000 con 100 ul por pozo y durante una hora. Al cabo de este tiempo, y después de un extensivo lavado, se procedió a revelar la reacción con la solución cromógena 4-cloro-1-naftol (Immunopure Rockford, Illinois, USA. No 34010)

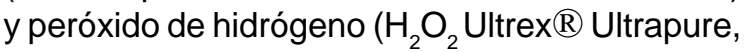
JT Baker, Phillipsburg, NJ, USA). La solución se dejó reaccionar por espacio de 20 minutos, al cabo de los cuales la reacción se paró con agua destilada y se quitó la membrana del equipo. Esta membrana se dejó en lavado en agua destilada y en agitación antes de proceder a secarla, para así leer los pozos positivos o negativos de cada membrana.

La lectura se determinó de acuerdo al cambio de color presentado tanto en las muestras bajo evaluación como en los controles negativos, en los positivos y en los pozos blanco que determinaron la coloración de fondo de la prueba.

\section{Protocolo para la obtención del extracto antigénico de glicoproteínas}

En el caso de la EITB, los antígenos se obtuvieron en el Laboratorio de Teniosis y Cisticercosis de la UNAM de México y consistieron en 7 glicoproteínas semipurificadas, GP50, GP42-39, GP24, GP21, GP18, GP14 y GP13 (las letras GP indican la naturaleza glicoproteica de estos antígenos y el número denota su peso molecular en kd), de acuerdo a lo descrito por Tsang et al. (7). El protocolo se siguió con algunas 
Cuadro 1. Resultados de la evaluación de la ELISA de punto. Parámetros de sensibilidad, especificidad y valores pronósticos positivo y negativo, con intervalos de confianza de $95 \%$ de acuerdo con el tipo de muestra.

\begin{tabular}{|c|c|c|c|c|c|c|c|c|}
\hline \multirow[t]{2}{*}{ Muestra } & \multicolumn{2}{|c|}{ EITB $^{1}(+)$} & \multicolumn{2}{|c|}{ EITB (-) } & \multirow{2}{*}{$\begin{array}{c}\text { S (\%) } \\
\text { (IC95\%) }\end{array}$} & \multirow{2}{*}{$\begin{array}{l}\text { E (\%) } \\
\text { (IC95\%) }\end{array}$} & \multirow[t]{2}{*}{ VPP } & \multirow[t]{2}{*}{ VPN } \\
\hline & $\operatorname{EP}^{2}(+)$ & EP (-) & $\mathrm{EP}(+)$ & $E P(-)$ & & & & \\
\hline Suero & 41 & 4 & 0 & 37 & $91,1(89,9-92,3)$ & $100(98,7-100)$ & $100(98,7-100)$ & $90,2(89,0-91,5)$ \\
\hline Plasma & 35 & 6 & 9 & 55 & $85,37(84,1-86,7)$ & $85,9(85,1-86,8)$ & $79,55(78,3-80,8)$ & $90,2(89,3-91,0)$ \\
\hline LCR & 12 & 11 & 0 & 17 & $52,1 \quad(49,88-54,47)$ & $100(97,1-100)$ & $100(95,18-100)$ & $60,7(58,8-62,6)$ \\
\hline $\begin{array}{l}\text { Total } \\
\text { Sueros }\end{array}$ & 88 & 21 & 9 & 109 & $80,7(80,2-81,2)$ & $92,4(91,9-92,8)$ & $90,7(90,2-91,3)$ & $83,9(83,4-84,3)$ \\
\hline $\begin{array}{l}\text { Población } \\
\text { endémica }\end{array}$ & 7 & 5 & 0 & 651 & $58,33(54,0-62,7)$ & $100(99,9-100)$ & $100(92,9-100)$ & $99,2(99,1-99,3)$ \\
\hline
\end{tabular}

${ }^{1}$ EITB: inmunoelectrotransferencia ${ }^{2} \mathrm{EP}$ : ELISA de punto

modificaciones, entre las que se cuenta la no utilización de método de deslipidización alguno para la preparación de las glicoproteínas. En resumen, estas glicoproteínas se obtuvieron por cromatografía de afinidad luego de su paso por una columna de lectina de lenteja-sefarosa ${ }^{\circledR}$. Una vez purificados los antígenos, y luego de haber determinado su concentración proteica por el método de Bradford (12), se les distribuyó en alícuotas y se les almacenó a $-80^{\circ} \mathrm{C}$ hasta su uso en la ejecución del EITB, según procedimiento descrito por Tsang et al. (7).

\section{Desarrollo del EITB}

En términos generales, la técnica se realizó utilizando minigeles en gradiente de $5 \%$ a $15 \%$ de solución de poliacrilamida (Bio Rad Laboratories, Richmond CA, USA, referencia 1610146), y anti-inmunoglobulina $G$ anti- humana (A-8792, Sigma St. Louis, MO, USA) marcada con peroxidasa como conjugado. Posteriormente, con el fin de revelar las reacciones antígenoanticuerpo producidas frente a los sueros, se empleó como reveladores el sistema peróxido de hidrógeno/3,3' diaminobenzidina $\left(\mathrm{H}_{2} \mathrm{O}_{2} / D A B\right.$, Aldrich Chemical Company, Inc. Milwaukee, WI, USA), o en otros casos, el sistema peróxido de hidrógeno/3,3', 5,5' tetrametilbenzidina $\left(\mathrm{H}_{2} \mathrm{O}_{2} /\right.$ $T M B$, Kirkegaard \& Perry Laboratories, Gaithersburg, MA, USA). El criterio de positividad se determinó, según lo recomendado, por la reacción de los sueros con al menos una de las 7 fracciones antigénicas (GP 50, 42-39, 24, 21, 18, 14 y 13). Las muestras que reaccionaron con más de tres bandas diagnósticas fueron utilizadas para conformar el grupo de positivos con el que se realizó la validación de la prueba de EP.

\section{Análisis estadístico}

El análisis estadístico se realizó con base en el registro de los datos en una tabla de doble entrada, utilizando el programa EPIDAT 3.0 (SERGAS, Xunta de Galicia, OPS, OMS) (14). Con base en esto se calcularon la sensibilidad, la especificidad y los valores pronósticos positivo y negativo de la prueba con sus intervalos de confianza de $95 \%$. Se tomó como prueba de oro el EITB para hacer los cálculos de sensibilidad y especificidad.

\section{Resultados}

Se estudiaron 933 muestras de suero, plasma o LCR de pacientes con cisticercosis, controles sanos, individuos con otras parasitosis o pertenecientes a un estudio de seroprevalencia de cisticercosis. En el cuadro 1 se relacionan los resultados de sensibilidad y especificidad y los valores predictivos positivos y negativos obtenidos para el EP comparado con el EITB, y según la muestra utilizada para realizar la prueba.

Después de determinar los parámetros de sensibilidad y especificidad del EP para el diagnóstico de la cisticercosis en los grupos de estudio establecidos, se evaluó la utilidad de la prueba para determinar la seroprevalencia de cisticercosis en una población endémica colombiana en la que $1,81 \%(12 / 663)$ de las personas presentó anticuerpos para $T$. solium con la EITB. EI EP presentó una sensibilidad de $58,3 \%$ (IC95\%: 54,0\% a 62,6\%) y una especificidad de 100\% (IC95\%: $99,9 \%$ a $100,0 \%$ ) con un valor 
diagnóstico positivo de 100\% (IC95\%: 92,9\% a $100,0 \%$ y un valor diagnóstico negativo de $99,2 \%$ (IC 95\%: 99,2\% a 99,3\%).

\section{Discusión}

El amplio espectro de los síntomas de la cisticercosis hace difícil realizar un diagnóstico con base únicamente en la presentación clínica; de ahí que los métodos imaginológicos e inmunodiagnósticos sean un complemento útil $(15,16)$.

A pesar de la utilidad diagnóstica de la TC y la $\mathrm{RM}$, su uso rutinario es limitado debido al alto costo de los equipos, lo cual determina que su disponibilidad sea escasa y que no se puedan aplicar a estudios epidemiológicos en áreas endémicas $(6,15,17)$. Los métodos inmunodiagnósticos para detectar anticuerpos en suero y LCR, disponibles para el diagnóstico de cisticercosis, se usan para confirmar hallazgos de TC y RM.

Recientemente, diversos grupos de investigadores han realizado estudios con el fin de mejorar los métodos inmunodiagnósticos disponibles. Hasta hace unos años, en nuestro medio la prueba más empleada era la ELISA, reemplazada después por el EITB, que tiene una sensibilidad de hasta $98 \%$ y de especificidad de $100 \%$ (7) cuando los pacientes presentan más de un quiste.

En este estudio se estandarizó y se evaluó la prueba de EP utilizando parámetros semejantes a los reportados por otros investigadores en diferentes regiones del mundo (18-22). Cabe anotar que esta investigación registra el mayor número de muestras procesadas (933), ofrece comparación estadística entre diferentes muestras diagnósticas y evalúa la prueba no sólo a nivel de grupos de estudio predeterminados sino también a nivel de un grupo de población general.

Los diferentes estudios que han empleado el EP como prueba inmunodiagnóstica para cisticercosis han encontrado valores de sensibilidad y especificidad que varían aproximadamente entre $50 \%$ y $100 \%$ (18-22). Las diferencias de sensibilidad y especificidad reportadas frente a las obtenidas en este estudio pueden deberse a las diversas técnicas de preparación del antígeno, a las condiciones de ejecución propias de las pruebas, así como a la variabilidad existente en la composición antigénica de $T$. solium y cisticercos obtenidos de diferentes fuentes de una misma región o de regiones diferentes de un mismo país, hecho documentado por varios autores (23-26).

La más alta sensibilidad se obtuvo cuando se utilizó el suero sanguíneo como muestra para la realización del EP $(91,1 \%)$, con la ventaja adicional de que es un procedimiento invasivo de mínimo riesgo para su obtención, lo que no sucede con el LCR, que es un procedimiento invasivo de mayor riesgo y con el que, además, se obtuvo la mas baja sensibilidad $(52,2 \%)$.

La sensibilidad y especificidad obtenidas para el EP realizado con suero $(91,11 \%$ y $100 \%)$, y su alto valor pronóstico positivo (100\%) en el estudio de seroprevalencia, ofrecen una combinación ideal por ser una prueba diagnóstica de fácil ejecución y bajo costo en muestras de fácil obtención.

Las condiciones de ejecución de la prueba son aplicables a cualquier laboratorio de áreas endémicas, proporcionando un instrumento útil de diagnóstico para los laboratorios que carezcan de equipo o personal entrenado. Por su fácil implementación y la estabilidad de los reactivos utilizados, constituye el método de elección para fines de vigilancia epidemiológica y para la evaluación de programas de vigilancia y control de la teniosis-cisticercosis.

Si se compara la estimación de la prevalencia de la cisticercosis con el EITB $(1,8 \%)$ con la que se obtiene por medio del EP (1.05\%), se encuentra que la prevalencia de la enfermedad se subestima en $45 \%$. Esto es de esperarse debido a que los niveles de anticuerpos obtenidos en estudios poblacionales son bajos, pero las dificultades técnicas de otras pruebas diagnósticas como el EITB, y la mayor invasividad de los procedimientos para la obtención de las muestras, hacen del EP una prueba útil para el estudio epidemiológicos de campo.

Los resultados obtenidos para el EP realizado en suero de pacientes con cisticercosis la convierten en una prueba por la que debería optarse en los 
laboratorios clínicos de las instituciones de salud de baja complejidad que no cuentan con la infraestructura necesaria para la realización del EITB ni los equipos de imágenes necesarios para el estudio de pacientes con diagnóstico presuntivo de neurocisticercosis.

Es necesario emprender nuevos estudios que permitan validar la prueba con extractos antigénicos semipurificados o purificados, recombinantes o sintéticos, para así mejorar los niveles de sensibilidad obtenidos, tal como lo reportan otros investigadores (27-30). Un estudio (31) que compara el EP con el EITB usando un antígeno purificado para Parastrongylus cantonensis, concluye que las pruebas son comparables en sus niveles de sensibilidad y especificidad.

El EP validado con fuentes antigénicas obtenidas en Colombia constituye una herramienta diagnóstica útil y promisoria para la realización de estudios de cisticercosis en las áreas endémicas, que pueden servir de base a futuros estudios sobre control de la enfermedad.

\section{Conflicto de intereses}

No existe conflicto de intereses.

\section{Financiación}

Esta investigación fue financiada en parte por el Ministerio de Salud de Colombia y por la Dirección Seccional de Salud de Antioquia.

\section{Referencias}

1. Botero D, Restrepo M. Cisticercosis. En: Parasitosis humanas. 4a ed. Medellín: CIB editores; 2003.

2. Schantz PM, Wilkins PP, Tsang VCW. Immigrants, imaging, immunoblots: the emergence of neurocysticercosis as a significant public health problem. En: Scheld WM, Craig WA, Hughes JM, editors. Emerging Infections. Washington, D.C.: American Public Health Association 1998. p.213-42.

3. Sciutto E, Fragoso G, Fleury A, Laclette JP, Sotelo J, Aluja A et al. Taenia solium disease in humans and pigs: an ancient parasitosis disease rooted in developing countries and emerging as a major health problem of global dimensions. Microbes Infect 2000;2: 1875-90.

4. García HH, González AE, Evans CA, Gilman RH, Cysticercosis Working Group in Perú. Taenia solium cisticercosis. Lancet 2003;362:547-56.
5. Schantz PM. Taenia solium cysticercosis/taeniosis is a potentially eradicable disease: developing a strategy for action and obstacles to overcome. En: García HH, Martínez SM, editors. Taenia solium: Taeniasis/ Cysticercosis. Second edition. Lima: Ed. Universo; 1999. p.215-17.

6. Díaz JF, Verastegui M, Gilman RH, Tsang VC, Pilcher JB, Gallo C et al. Immunodiagnosis of human cysticercosis (Taenia solium): a field comparison of an antibody-enzyme-linked-immunosorbent assay (ELISA), an antigen-ELISA, and an enzyme-linkedimmuno-electrotransfer-blot (EITB) assay in Perú. Am J Trop Med Hyg 1992;46:610-5.

7. Tsang VC, Brand JA, Boyer AE. An enzyme-linked immunoelectrotransfer blot assay and glycoprotein antigens for diagnosing of human cysticercosis (Taenia solium). J Infect Dis 1989;159:50-9.

8. Palacio LG, Jiménez I, García HH, Jiménez ME, Sánchez JL, Noh $\mathbf{J}$ et al. Neurocysticercosis in persons with epilepsy in Medellín, Colombia. Epilepsia 1998;39:1334-9.

9. García HH, González AE, Gilman RH, Palacios LG, Jiménez I, Rodríguez $\mathbf{S}$ et al. Short report: transient antibody response in Taenia solium infection in field conditions - a major contribution to high seroprevalence. Am J Trop Med Hyg 2001;65:31-2.

10. Agudelo Flórez P, Palacio LG. Prevalencia de anticuerpos para Taenia solium en humanos y cerdos en una zona endémica colombiana. Rev Neurol 2003; 36:706-9.

11. Grogl M, Estrada JJ, Macdonald G, Kunhn RE. Antigen-antibody analyses in neurocysticercosis. J Parasitol 1985;71:433-42.

12. Bradford MM. A rapid and sensitive method for the quantification of microgram quantities of protein utilizing principle of protein dye binding. Anal Biochem 1976; 72:248-54.

13. Cardona-Castro N, Agudelo-Florez P. Immunoenzymatic dot-blot test for the diagnosis of enteric fever caused by Salmonella typhi in an endemic area. Clin Microbiol Infect 1998;4:64-9.

14. Riegelman RK, Hirsch RP. Cómo estudiar un estudio y probar una prueba: lectura crítica de la literatura médica. Publicación Científica 531. 2 $2^{\mathrm{a}}$ edición. Washington, D.C.: Organización Panamericana de la Salud; 1992. p.260.

15. García HH, Herrera G, Gilman RH, Tsang VC, Pilcher JB, Díaz JF et al. Discrepancies between cerebral computed tomography and western blot in the diagnosis of neurocysticercosis. Am J Trop Med Hyg 1994;50: 152-57.

16. Flisser A, Plancarte A, Avila G. Application of diagnostic methods for cysticercosis and taeniosis to epidemiological studies. En: Garcia HH, Martínez SM, 
editors. Taenia solium: taeniasis/cysticercosis. Second edition. Lima: Ed. Universo; 1999. p.40-52.

17. Feldman $\mathbf{M}$, Plancarte $\mathbf{A}$, Sandoval $\mathbf{M}$, Wilson $\mathbf{M}$, Flisser A. Comparison of two assays (EIA and EITB) and two samples (saliva and serum) for the diagnosis of neurocysticercosis. Trans R Soc Trop Med Hyg 1990;84:559-62.

18. Plancarte A, Fexas M, Flisser A. Reactivity in ELISA and dot blot of purified GP24, an immunodominant antigen of Taenia solium, for the diagnosis of human neurocysticercosis. Int J Parasitol 1994;24:733-8.

19. Vaz JA, Ferreira AW. Imunodiagnóstico da neurocisticercose: teste imunoenzimático com antígenos quimicamente ligados a suportes para pesquisa de anticorpos em soro e líquido cefalorraquiano. Rev Inst Med Trop Sao Paulo 1988; $30: 1-9$.

20. Vaz AJ, Ferreira AW, Camargo ME, Nakamura PM, Camargo ED. Dot-ELISA for detection of antiCysticercus cellulosae antibodies in human cerebrospinal fluid using a new solid-phase (Resintreated polyester fabrics). Preliminary report. Rev Inst Med Trop Sao Paulo 1990;32:355-9.

21. Liu YS, Du WP, Wu ZX. Dot-immunogold-silver staining in the diagnosis of cysticercosis. Int J Parasitol 1996; 26:127-9.

22. Biswas R, Parija SC, Narayan SK. Dot-ELISA for the diagnosis of neurocysticercosis. Rev Inst Med Trop Sao Paulo 2004;46:249-52.

23. Vega R, Pinero $D$, Ramanankandrasana $B$, Dumas M, Bouteille B, Fleury A et al. Population genetic structure of Taenia solium from Madagascar and Mexico: implications for clinical profile diversity and immunological technology. Int J Parasitol 2003;33: 1479-85.
24. Maravilla $\mathbf{P}$, Souza V, Valera A, Romero-Valdovinos M, López-Vidal Y, Domínguez-Alpizar JL, et al. Detection of genetic variation in Taenia solium. J Parasitol 2003;89:1250-4.

25. Maravilla $\mathbf{P}$, Valera A, Souza V, Martínez-Gordillo M, Flisser A. Isozyme analysis of Taenia solium isolates from Mexico and Colombia. Mem Inst Oswaldo Cruz 2003; 98:1049-50.

26. Ito A, Yamasaki H, Nakao M, Sako Y, Okamoto M, Sato MO, et al. Multiple genotypes of Taenia soliumramifications for diagnosis, treatment and control. Acta Trop 2003;87:95-101.

27. Verastegui M, Gilman RH, García HH, González AE, Arana Y, Jeri C et al. Prevalence of antibodies to unique Taenia solium oncosphere antigens in taeniasis and human and porcine cysticercosis. Am J Trop Med Hyg 2003;69:438-44.

28. Fleury A, Beltrán C, Ferrer E, Garate T, Harrison LJ, Parkhouse RM, et al. Application of synthetic peptides to the diagnosis of neurocysticercosis. Trop Med Int Health 2003;8:1124-30.

29. Hancock K, Pattabhi S, Greene RM, Yushak ML, Williams F, Khan A et al. Characterization and cloning of GP50, a Taenia solium antigen diagnostic for cysticercosis. Mol Biochem Parasitol 2004;133:115-24.

30. Levine MZ, Calderon JC, Wilkins PP, Lane WS, Asara JM, Hancock $\mathrm{K}$ et al. Characterization, cloning, and expression of two diagnostic antigens for Taenia solium tapeworm infection. J Parasitol 2004;90:631-8.

31. Eamsobhana $\mathbf{P}$, Yoolek A, Punthuprapasa $\mathbf{P}$, Suvouttho S. A dot-blot ELISA comparable to immunoblot for the specific diagnosis of human parastrongyliasis. J Helminthol 2004;78:287-91. 